\title{
Towards a Perfect Voting System
}

\author{
Elena Karachaliou* , Alex Brekoulakis, John Stratoulias \\ The Moraitis School, GR-15452, Athens, Greece.
}

How to cite this paper: Elena Karachaliou, Alex Brekoulakis, John Stratoulias. (2020) Towards a Perfect Voting System. Journal of Applied Mathematics and Computation, 4(4), 241-248.

DOI: $10.26855 /$ jamc.2020.12.015

Received: October 20, 2020

Accepted: November 28, 2020

Published: December 18, 2020

*Corresponding author: Elena Karachaliou, The Moraitis School, GR-15452, Athens, Greece. Email: kar.elena99@gmail.com

\begin{abstract}
Choosing a voting system may seem a simple-looking procedure, however, it has to take into account many different social and political parameters as well as some mathematical contradictions. Thus, whether a voting system is considered fair or not is a debate that has been concerning researchers for decades. In this paper, we examine what makes a voting system fair, successful and effective. In order to achieve this, we present some of the most well-known voting systems and explain their mechanisms. Then, we present some basic mathematical properties-criteria of social choice procedures. After all, the satisfaction or not of such properties is what determines the fairness of a voting system. The question that arises is: "Can a voting system fulfill all these properties?” We show that all voting systems fail to satisfy some of the properties and we finally mention the impossibility theorems that prove that there is no social choice procedure that satisfies all properties, for example, the Gibbard-Satterthwaite theorem which can be practically applied. However, by examining the legitimacy of such claims, we discover voting paradoxes that arise in many hypothetical election scenarios, such as contradictions between some of the set criteria, and also scenarios in which it is impossible to fulfill some of these criteria, the existence of which seems to dictate that there is no perfect voting system. Or is there?
\end{abstract}

\section{Keywords}

Voting System, Elections, Criteria of Voting Systems, Contradictions and Paradoxes of Voting Systems, Fairness of Voting Systems

\section{Introduction}

Elections are possibly the most important part of democracy, since elections constitute the indirect way through which citizens govern a country, which makes an electoral/voting system also very important. Such a system is usually defined as the procedure through which votes are converted to seats in a country's parliament, a company's board, etc. However, in this paper, we are going to examine voting systems in the sense of the procedure through which the votes are actually casted and valued, e.g., if the voter can choose from one or more candidates, if the voters grade the candidates with points, etc.

Since a voter's preferences remain the same no matter which system is used, the choice of a voting system is perceived as a trivial matter that has practically no effect on the outcome. However, as we demonstrate below, this choice will not only largely affect the outcome, but it will also define whether the election outcome is fair, representative and, consequently, democratic. Also, a major factor is the satisfaction or non-satisfaction of a plethora of criteria that a voting system needs to fulfil. Some of the most commonly accepted criteria are also demonstrated below, in the second section of this paper.

As made apparent by the above statements, the making of a voting system is a complex procedure. It requires elaborate work, testing and reconsideration, so that the final product, by satisfying the soon-to-be-ment criteria, cannot be subject to manipulation, contradictions and misrepresentation in any scenario. However, in the third part we present observations and theorems which claim that no voting system can satisfy all, or certain sets of, criteria, dictating that absolute fairness and accuracy in representation can never be achieved. Moreover, sometimes even logical contradictions can occur, meaning 
that results can go against common logic at first glance. In this case, such a phenomenon is called a "paradox" and the examination of such phenomena comprises the final part of this presentation.

\section{Voting Systems}

In this section, we mention some of the most well-known voting systems. These are the plurality, Condorcet's method, Borda count, first past the post system, single transferable vote and range voting.

\subsection{Plurality}

Plurality is divided into two categories: relative and absolute majority. The relative majority declares as a winner the candidate with the largest number of votes. However, it is not necessary for the winner to have more than half of the votes. According to the absolute majority, the winner is obligated to have more than half of the votes otherwise a two-round system or a ranked voting system is applied. The two-round system, which is called plurality with runoff, is applied in many countries such as France, Portugal, Brazil, Cyprus etc. [1].

For example, if the voters' preferences are as shown in Table 1 it means that 4 voters (the first column) would prefer as a winner candidate A, their second preference would be candidate B, their third one candidate C and last one candidate D. 3 other voters (the second column) would vote for candidate B as the winner, C would be their second choice and so on...

According to plurality, taking under consideration the first preferences of the voters, the winner should be candidate $\mathrm{D}$ as he has the absolute majority of the votes (9 out of 16 ).

Table 1. Voters' preferences for plurality

\begin{tabular}{cccc}
\hline 4 voters & 3 voters & 5 voters & 4 voters \\
\hline A & B & D & D \\
B & C & A & B \\
C & A & B & C \\
D & D & C & A \\
\hline
\end{tabular}

\subsection{Condorcet's method:}

During the $18^{\text {th }}$ century, Marquis de Condorcet, a French philosopher, mathematician and political scientist, developed another voting system which suggested a pairwise comparison between the alternatives. That is to say that doing one-on-one contests between the alternatives we ultimately reach to the final winner which is the one who wins all the other alternatives on these one-to-one contests [3, p. 6].

For example, four candidates are ranked by 16 voters as shown in Table 1. First, we compare A and B, then B and C and finally $A$ and $C$. When we compare candidates $A$ and $B$ we ignore the other candidates $(C)$ and we have $A$ with $4+5=9$ votes and B with 3+4=7 votes. Thus candidate A wins candidate B. Following the same procedure for the rest of the candidates we get:

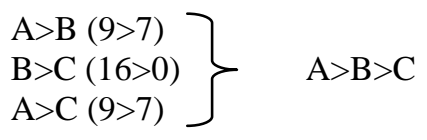

According to Condorcet he winner should be candidate A.

\subsection{Borda Count:}

During the $18^{\text {th }}$ century, Jean-Charles de Borda, a French mathematician, physicist, political scientist and sailor developed a new voting system. According to this voting system, each voter sets an order of preference for the alternatives. Then, we assign points to every alternative depending on their position. For example for the 4st position we assign 1 point, for the 3rd 2 points etc. [3, pp. 6-7]. The alternative with the most points wins. Its "disadvantage" is that it does not always satisfy the Condorcet's winner criterion, the Plurality criterion and the Independence of Irrelevant Alternatives. The advantages of this system are that it satisfies the Monotonicity and Condorcet's Loser, as well as the fact that it takes regard to the comparative preferences of the voters towards candidates. The Borda count is used for the election of two ethnic-minority members of the National Assembly of Slovenia, the Parliament of Nauru, the presidential elections in Kiribati and various private organisations and awards, such as the NBA's Most Valuable Player award and the Eurovision Song Contest [1]. 
Table 2. Voters' preferences for Borda Count

\begin{tabular}{cccc}
\hline $\mathbf{4}$ voters & 3 voters & $\mathbf{5}$ voters & $\mathbf{4}$ voters \\
\hline A [3] & B [3] & D [3] & D [3] \\
B [2] & C [2] & A [2] & B [2] \\
C [1] & A [1] & B [1] & C [1] \\
D [0] & D [0] & C [0] & A [0] \\
\hline
\end{tabular}

If for example the preference lists of 16 voters are as shown in Table 2 according to the Borda count the results are:

$\mathrm{A} \rightarrow 4 * 3+3 * 1+5 * 2+4 * 0=25$

$\mathrm{B} \rightarrow 4 * 2+3 * 3+5 * 1+4 * 2=30$

$\mathrm{C} \rightarrow 4 * 1+3 * 2+5 * 0+4 * 1=14$

$\mathrm{D} \rightarrow 4 * 0+3 * 0+5 * 3+4 * 3=27$

As a result, the winner according to Borda should be candidate B with 30 votes.

\subsection{Coombs’ Method:}

Clyde Coombs, a psychologist in the $20^{\text {th }}$ century, suggested another voting system where the voters rank all of the candidates on their ballot. If at any time one candidate is ranked first (among non-eliminated candidates) by an absolute majority of the voters, that candidate wins. Otherwise, the candidate ranked last (again among non-eliminated candidates) by the largest number of voters is eliminated. In other words, under instant-runoff voting, the candidate ranked first (among non-eliminated candidates) by the fewest voters is eliminated.

Table 3. Voters' preferences for Coombs

\begin{tabular}{cccc}
\hline 4 voters & 3 voters & $\mathbf{5}$ voters & 4 voters \\
\hline A & B & D & B \\
B & C & A & A \\
C & A & B & C \\
D & D & C & D \\
\hline
\end{tabular}

For example, as shown in Table 3 no candidate has the absolute majority (at least 9 votes) so we eliminate the candidate ranked last by most of the voters, candidate $\mathrm{D}$. Then the next candidate to be eliminated is candidate $\mathrm{C}$ (13 out of 16 last votes). Finally, candidate B is eliminated with 9 votes which leaves as the winner candidate A.

\subsection{First past the post system (FPTP):}

In FPTP, voters cast a single preference vote, meaning they only vote for their top preference, and the candidate that has a simple majority rather than an absolute one is elected. This means that they do not need to have over $50 \%$, instead just more than any other candidate. A downside of this voting system is that it essentially invites for strategic voting. This happens because supporters of minority parties or candidates, knowing there is little chance of their top preference to be elected, vote for a major party or candidate close to their preference so that the latter's percentage surpasses that of other major parties which are farther away from the voter's preference. This system is applied in Great Britain, Australia, Canada and India [1].

Applying the FPTP system to Table 1 the results are:

$$
\begin{aligned}
& \mathrm{A} \rightarrow 4 \text { votes } \\
& \mathrm{B} \rightarrow 3 \text { votes } \\
& \mathrm{C} \rightarrow 0 \text { votes } \\
& \mathrm{D} \rightarrow 9 \text { votes }
\end{aligned}
$$

Therefore, candidate $\mathrm{D}$ is the winner.

\subsection{Single Transferable Vote (STV):}

The STV is a more specific form of the FPTP and it is quite interesting as it elects more than one winner. For this system, a minimum number of votes is set (the limit) and the voters are obliged to create their individual preference lists. Then, by adding the votes of each list for every voter we check whether any candidate has surpassed the limit or not. If he has then he 
is immediately a winner. Afterwards, the difference of his votes minus the limit is given to the second choice of the voters. If someone else surpasses the limit the same procedure is followed. If at some point there is no one that reaches the limit, the votes of the candidate with the less votes are shared at the second choice of these specific voters. These two procedures are repeated again and again as many times as needed [7]. The STV is applied in Ireland, Malta, United States, Australia and other government elections [1].

\subsection{Range voting (also known as score voting):}

In the RV system, voters grade candidates using a numerical score (usually 1 to 10 or 1 to 100) for each. The candidate with the highest average wins. This system is a single-winner voting system which means that only one candidate can be elected. Some of its advantages are that it reflects the preference of the voters better and the results are easy to understand and more accurate than other electoral systems.

A form of the system was used in some elections in ancient Sparta in which the candidate whose name was shouted louder was elected.

The ballot would look like this (Figure 1):

\begin{tabular}{|c|c|c|}
\hline $\begin{array}{l}\text { Governor } \\
\text { Candidates }\end{array}$ & $\rightarrow$ & $\begin{array}{l}\text { Score each candidate by bubbling a } \\
\text { number ( } 0 \text { is worst; } 9 \text { is best) }\end{array}$ \\
\hline 1: Candidate A & $\rightarrow$ & \\
\hline 2: Candidate B & $\rightarrow$ & \\
\hline 3: Candidate C & $\rightarrow$ & \\
\hline
\end{tabular}

Figure 1. Voters’ preferences for range voting.

\section{Criteria}

When creating or seeking for a voting system, there is a plethora of criteria and properties desirable for a voting system to fulfill. However, as contradictions between criteria we are going to examine goes to show, and as demonstrated by the Gibbard-Sattherwaite theorem, Arrow's impossibility theorem and other mathematical theorems, it is probably impossible for a voting system to not violate at least one criterion or property. This will be further examined in the next section. Because of this, we need to prioritize and rank criteria, and then choose our voting system based on our most highly preferred ones. There are three (3) criteria ranked at the top by most sources, since they follow common sense, their fulfillment is rather easy to achieve, and they are intercompatible. These are the Pareto condition, the transitivity and the regard to second choice. However, there are some other criteria we are going to analyze as well.

\section{1) The Pareto condition:}

A social choice procedure satisfies the Pareto condition when in case everyone prefers candidate B to C, then C is not considered the social choice or even is among the social choices in a tie, thus is not elected. For example, as shown in Table 4, C should never be elected since candidate B is preferred for all voters [3, pp. 11-12].

Table 4. Voters' preferences; the Pareto condition

\begin{tabular}{cccc}
\hline 4 voters & 3 voters & 5 voters & 4 voters \\
\hline A & B & D & D \\
B & C & A & B \\
C & A & B & C \\
D & D & C & A \\
\hline
\end{tabular}

\section{2) Transitivity:}

Same as in the mathematical property of transitivity. The transitivity criterion dictates that if the majority of voters 
prefers $A$ to $B$ and $B$ to $C$, then $A$ is ranked higher than $C$. Mathematically, if $A>B$ and $B>C$, then $A>C$ (that is similar to the transitivity property in Algebra).

\section{3) Regard to second choice:}

A voting system needs to not depend only on the voters' first choice. In other words, there needs to exist a scenario and a set of votes in which the second choice of the voters influences the outcome. A characteristic example of a system that does not satisfy this criterion is the Plurality.

\section{4) The plurality criterion:}

Plurality dictates that when a candidate has over $50 \%$ of the votes, then he/she should always be elected.

\section{5) The Condorcet criterion:}

A candidate in an election who would defeat every other candidate in a one-on-one or pairwise comparison is said to be a Condorcet winner. A voting system is said to satisfy the Condorcet criterion when it always elects the Condorcet winner (when there is one) [2].

\section{6) Condorcet's loser:}

Condorcet's loser is basically the opposite of Condorcet's winner. If A loses against all other candidates in a one-on-one comparison, then A is a Condorcet's loser and should not be elected.

\section{7) Coombs' criterion:}

According to Coombs, when a candidate is ranked last by the majority of voters, that candidate should not be elected. It is widely regarded as one of the systems that depict social choice the best, even though the results at first may seem irrational. This is because a candidate might be ranked last by all voters except the group that voted.

\section{8) Monotonicity:}

\section{Table 5. Voters’ preferences; monotonicity}

\begin{tabular}{ccc}
\hline Voter 1 & Voter 2 & Voter 3 \\
\hline B & A & A \\
A & D & C \\
C & C & B \\
D & B & D \\
\hline
\end{tabular}

A voting system is characterized as monotone or monotonic when in a scenario that $\mathrm{A}$ is the social choice, if someone changes his/her preference list in a way favorable to $A$ then $A$ should still remain the social choice. For example if in a set of votes that elects $\mathrm{A}$, someone ranks $\mathrm{B}>\mathrm{A}>\mathrm{C}>\mathrm{D}$, and they change it to $\mathrm{A}>\mathrm{B}>\mathrm{C}>\mathrm{D}$, then $\mathrm{A}$ should still be elected, as depicted in Table 6 [3, p. 12].

Table 6. Voters' preferences; monotonicity

\begin{tabular}{ccc}
\hline Voter $\mathbf{1}$ & Voter $\mathbf{2}$ & Voter 3 \\
\hline A & A & A \\
B & D & C \\
C & C & B \\
D & B & D \\
\hline
\end{tabular}

\section{9) Independence of irrelevant alternatives:}

The independence of irrelevant alternatives criterion dictates that when in a social choice procedure someone changes his preference list but he does not change the relative positions between two candidates then the relationship between them 
remains the same. For example, if someone ranks $B>A>C>D$, so that $A>C$, then, if they change their vote to $D>A>C>B$, it should still be $\mathrm{A}>\mathrm{C}[3$, pp. 12-13].

Table 7. Voters' preferences; independence of irrelevant alternatives

\begin{tabular}{ccc}
\hline Voter 1 & Voter 2 & Voter 3 \\
\hline B & A & A \\
\hline A & D & C \\
C & C & B \\
D & B & D \\
\hline
\end{tabular}

Table 8. Change in voters' preferences; independence of irrelevant alternatives

\begin{tabular}{ccc}
\hline Voter $\mathbf{1}$ & Voter $\mathbf{2}$ & Voter 3 \\
\hline D & A & A \\
A & D & C \\
C & C & B \\
B & B & D \\
\hline
\end{tabular}

\section{Contradictions and Paradoxes}

According to Mark Sainsbury a definition for the word "paradox" is "an unacceptable conclusion derived by apparently acceptable premises” or, by the Oxford dictionary definition, “a seemingly absurd or contradictory statement or position which when investigated may prove to be well founded or true”. The term seems to have derived from the Greek word "paradoxon" (Greek: $\pi \alpha \rho \alpha \dot{\delta} \delta \xi o v$ ) which means something contrary. The term probably appeared in the middle of the 16th century in Latin [5].

In this section, we are going to analyze and explain the contradiction between the Borda count, Condorcet's method and the plurality, the Condorcet's paradox and Arrow's theorem.

\subsection{Borda count contradicting Condorcet and Plurality}

The Borda count system and its derivatives do not always satisfy the Plurality criterion and the Condorcet's winner criterion. For example the voters' preference lists are as shown in Table 9.

Table 9. Borda's count, Condocet's method and plurality

\begin{tabular}{ccc}
\hline & Number of voters and preferences & \\
\hline 14 & 10 & 2 \\
A & C & D \\
B & B & B \\
C & D & C \\
D & A & A \\
\hline
\end{tabular}

Through the procedure of Plurality the winner is candidate A since he/she wins with 14 votes (absolute majority).

According to the Condorcet's method, the winner is again A because he/she wins every other alternative on all one-on-one contests: 


$$
\left.\begin{array}{l}
A>B(14>12) \\
B>C(16>10) \\
A>C(14>12)
\end{array}\right\} \quad A>B>C
$$

However, using the Borda count the points are:

A gets $14 * 3+10 * 0+2 * 0=42$ points

B gets $14 * 2+10 * 2+2 * 2=52$ points

C gets $14 * 1+10 * 3+2 * 1=46$ points

D gets $14 * 0+10 * 1+2 * 3=16$ points

$\mathrm{B}>\mathrm{C}>\mathrm{A}>\mathrm{D}$

We can clearly see that the winner is $B$.

For the same preference lists, we applied three procedures and we got two different results. This can be explained because the Borda count may violate the Plurality Criterion and the Condorcet's winner criterion [6].

\subsection{Condorcet's Paradox}

Table 10. Voters' preferences; condorcet’s paradox

\begin{tabular}{ccc}
\hline voter 1 & voter 2 & voter 3 \\
\hline A & B & C \\
B & C & A \\
C & A & B \\
\hline
\end{tabular}

Applying Condorcet's method (comparing the alternatives) the results are:

$\mathrm{A}>\mathrm{B}$

$\mathrm{B}>\mathrm{C}$

$\mathrm{C}>\mathrm{A}$

Then, it should be valid that $\mathrm{A}>\mathrm{B}>\mathrm{C}>\mathrm{A}$

This cyclic relationship between the alternatives prevents from concluding to a unique winner [4, pp. 15-22].

\subsection{Arrow's Theorem}

Kenneth Arrow won a Nobel Prize in Economics [10] for, among other achievements, the following theorem that he stated and proved:

A voting system is impossible to satisfy the following criteria at the same time:

1) There is no dictator (someone whose opinion overpowers all the other opinions )

2) If all the voters prefer A to B then at the final results A should be ranked higher than B (unanimity).

3) There is always only one winner/ there are no ties (universality).

4) It satisfies the Independence of Irrelevant Alternatives.

The problem is that Arrow defined a voting system as a system in which the voters classify the candidates. As a result Arrows definition limits the range of voting systems in which it is applied and for example does not consider Range Voting as a voting system even though it is [8].

\section{Conclusion}

In this paper, we have presented some well-known voting systems.

As demonstrated above, Arrow's theorem is only applied for a class of voting systems as described in its definition. This leaves the Gibbard-Satterthwaite theorem as the only valid theorem proving no voting system can satisfy a given set of criteria, other than the ones in Arrow.

The criteria that are set by the Gibbard-Satterthwaite theorem are as follows:

1) There is no dictator.

2) If everyone prefers $A$ to everyone else then $A$ is the unique winner.

3) The voting system is only based on the votes, not luck.

4) At least 3 voters participate.

5) The sincere and strategic vote is the same. That is to say that voting according to the real preference of the voter is also the best strategic option. 
Therefore, it seems that there cannot be any absolute fairness in existing or future voting systems [9].

Or can there?

On November 19, 2009, Forest W. Simmons constructed an electoral system that equilibrates the strategic vote with the sincere vote and also satisfied all the criteria that the Gibbard-Satterthwaite theorem set, making its results doubtable.

Since there is seemingly no formal referral of this system anywhere, it probably hasn't been looked into. Consequently, the practical application of this system looks distant. However, many researchers believe that in the future this system and its principles can be modified to make its real-life application possible, creating a new era of voting systems free of the limits set by the Gibbard-Satterthwaite theorem. Will we finally have the ultimate voting system? Only time can tell!

\section{Acknowledgments}

We would like to thank our supervisor, Kostis Andriopoulos, for his help and guidance for the completion of our project. Also, we are grateful to our school, The Moraitis School, for providing us the suitable conditions to work on this project. Finally, we would like to thank the Programme and Organizing Committees of Euromath 2015 for awarding us with the Third Prize on Group Presentations.

\section{References}

[1] www.electoral-reform.org.uk/.

[2] The Math Forum, Condorcet Criterion.

[3] http://mathforum.org/library/drmath/view/52276.html.

[4] Alan D. Taylor, \& Allison M. Pacelli. (2008). Mathematics and Politics, Springer, USA.

[5] Hannu Nurmi. (1999). Voting Paradoxes and How to Deal with Them, Springer, Berlin. http://www.oxforddictionaries.com/definition/english/paradox.

[6] Mathias Risse. (2005). Why the count de Borda cannot beat the Marquis de Condorcet, Springer-Verlag.

[7] Ace Project, The Single Transferable Vote (STV). http://aceproject.org/ace-en/topics/es/esd/esd02/esd02d/default

[8] Arrow's "impossibility" theorem—how can range voting accomplish the impossible? http://rangevoting.org/ArrowThm.html.

[9] The Gibbard-Satterthwaite theorem about honest \& strategic voting. http://rangevoting.org/GibbSat.html.

[10] The Sveriges Riksbank Prize in Economic Sciences in Memory of Alfred Nobel 1972. http://www.nobelprize.org/ nobel_prizes/economic-sciences/laureates/1972/. 